\title{
Acoustofluidics 1: Governing equations in microfluidics
}

\section{Bruus, Henrik}

\section{Published in:}

Lab on a Chip

Link to article, DOI:

$10.1039 / \mathrm{c} 1 \mathrm{lc} 20658 \mathrm{c}$

Publication date:

2011

\section{Document Version}

Publisher's PDF, also known as Version of record

Link back to DTU Orbit

\section{Citation (APA):}

Bruus, H. (2011). Acoustofluidics 1: Governing equations in microfluidics. Lab on a Chip, 11(22), 3742-3751. https://doi.org/10.1039/c1lc20658c

\section{General rights}

Copyright and moral rights for the publications made accessible in the public portal are retained by the authors and/or other copyright owners and it is a condition of accessing publications that users recognise and abide by the legal requirements associated with these rights.

- Users may download and print one copy of any publication from the public portal for the purpose of private study or research.

- You may not further distribute the material or use it for any profit-making activity or commercial gain

- You may freely distribute the URL identifying the publication in the public portal

If you believe that this document breaches copyright please contact us providing details, and we will remove access to the work immediately and investigate your claim 


\title{
Acoustofluidics 1: Governing equations in microfluidics
}

\author{
Henrik Bruus
}

DOI: $10.1039 /$ c1lc20658c

\begin{abstract}
In Part 1 of the thematic tutorial series "Acoustofluidics - exploiting ultrasonic standing waves forces and acoustic streaming in microfluidic systems for cell and particle manipulation", we establish the governing equations in microfluidics. Examples of basic flow solutions are presented, and equivalent circuit modeling for determining flow rates in microfluidic networks is introduced.
\end{abstract}

\section{Introduction}

Microfluidics deals with the flow of fluids and suspensions in channels of sub-millimetre-sized cross-sections under the influence of external forces. ${ }^{1-6}$ Here, viscosity

Department of Micro- and Nanotechnology, Technical University of Denmark DTU

Nanotech, Building 345 B, DK-2800, Kongens Lyngby, Denmark

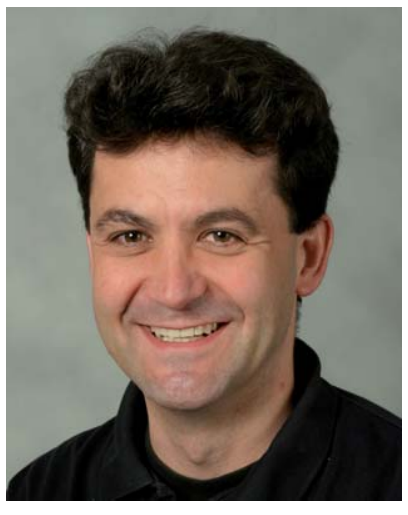

Prof. Henrik Bruus received his B.Sc. in mathematics and physics from the University of Copenhagen in 1984 and his M.Sc. and Ph.D. degrees in physics from the Niels Bohr Institute, University of Copenhagen in 1986 and 1990, respectively. He was postdoctoral fellow at Nordic Institute of Theoretical Physics 1990-92, Yale University 1992-94 and CNRS Grenoble 1994-96. He returned to the Niels Bohr Institute as an associate professor 1997-2001, before he joined the faculty at DTU Nanotech, Technical University of Denmark in 2002. He was promoted full professor there in 2005. He has (co)authored more than 90 peer-reviewed journal papers on condensed matter physics and microfluidics, as well as 110 peer-reviewed conference contributions and 2 monographs, the latest being "Theoretical Microfluidics", Oxford University Press (2008). on-chip acoustophoretic separation devices. ${ }^{17,18}$ Many different biotechnical applications of acoustophoresis have subsequently emerged including cell trapping, ${ }^{19-21}$ plasmapheresis, ${ }^{22}$ forensic analysis, ${ }^{23}$ food analysis, ${ }^{24}$ cell sorting using surface acoustic waves, ${ }^{25}$ cell synchronization, ${ }^{26}$ and cell differentiation. $^{27} \quad$ Furthermore, substantial advancements in understanding the fundamental physics of microsystems acoustophoresis have been achieved through full-chip imaging of acoustic resonances, ${ }^{28}$ surface acoustic wave generation of standing waves, ${ }^{29}$ multiresonance chips, ${ }^{30}$ advanced frequency control, ${ }^{31,32}$ on-chip integration with magnetic separators, ${ }^{33}$ acoustics-assisted microgrippers ${ }^{34}$ in situ force calibration, ${ }^{35}$ and automated systems. ${ }^{36}$

In Part 1 of the Acoustofluidics Tutorial Series we study the governing equations in microfluidics formulated in terms of the classical continuum field description of velocity $\boldsymbol{v}$, pressure $p$, and density $\rho$. We also present some of the basic flow solutions as well as the equivalent circuit modeling useful for predicting the flow rates through networks of microfluidic channels.

\section{The basic continuum fields}

In the following we use the so-called Eulerian picture of the continuum fields, where the spatial coordinates $r$ are fixed in space, and where we observe how the fields evolve in time at these points. Consequently, the position $r$ and the time $t$ are independent variables. The Eulerian picture is illustrated by the velocity field in Fig. 1. In general, the value of any field variable $F(\boldsymbol{r}, t)$ is defined as the average 

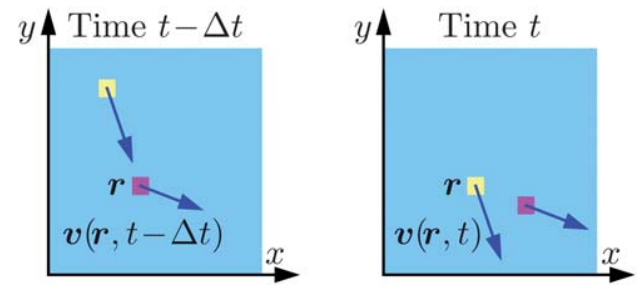

Fig. 1 The Eulerian picture: the spatial coordinates $r$ do not follow the flow. Instead, the velocity field $v$ at the fixed point $r$ is defined by the molecules in the purple region at time $t-\Delta t$, and by those in the yellow region at time $t$.

value $\left\langle F_{\mathrm{mol}}\left(\boldsymbol{r}^{\prime}, t\right)\right\rangle$ of the corresponding molecular quantity for all the molecules contained in some liquid particle of volume $\Delta V(\boldsymbol{r})$ around $\boldsymbol{r}$ at time $t$,

$$
F(\boldsymbol{r}, t)=\left\langle F_{\mathrm{mol}}\left(\boldsymbol{r}^{\prime}, t\right)\right\rangle_{\boldsymbol{r}^{\prime} \in \Delta V(\boldsymbol{r})}
$$

If we for brevity let $m_{i}$ and $\boldsymbol{v}_{i}$ be the mass and the velocity of molecule $i$, respectively, and furthermore let $i \in \Delta V$ stand for all molecules $i$ present inside the volume $\Delta V(\boldsymbol{r})$ at time $t$, then the definition of the density $\rho(\boldsymbol{r}, t)$ and the velocity field $\boldsymbol{v}(\boldsymbol{r}, t)$ can be written as

$$
\begin{gathered}
\rho(\boldsymbol{r}, t) \equiv \frac{1}{\Delta V} \sum_{i \in \Delta V} m_{i}, \\
\boldsymbol{v}(\boldsymbol{r}, t) \equiv \frac{1}{\rho(\boldsymbol{r}, t) \Delta V} \sum_{i \in \Delta V} m_{i} v_{i} .
\end{gathered}
$$

Here, we have introduced the sign " $\equiv "$ for "equal-to-by-definition sign". Notice how the velocity is defined through the more fundamental concept of momentum.

The dependent field variables in microfluidics can be scalars (such as density $\rho$, viscosity $\eta$, pressure $p$, temperature $T$, and free energy $\mathcal{F}$ ), vectors (such as velocity $\boldsymbol{v}$, current density $\boldsymbol{J}$, pressure gradient $\nabla p$, force density $\boldsymbol{f}$, and electric field $\boldsymbol{E}$ ), and tensors (such as stress tensor $\boldsymbol{\sigma}$ and velocity gradient $\boldsymbol{\nabla} \boldsymbol{v}$ ).

\section{Mathematical notation}

The mathematical treatment of microfluidic problems is complicated due to the presence of several scalar, vector and tensor fields and the non-linear partial differential equations that govern them. To facilitate the treatment some simplifying notation is called for. We follow ref. 4.

First, a suitable co-ordinate system must be chosen. We shall mainly work with Cartesian co-ordinates $(x, y, z)$ with corresponding basis vectors $\boldsymbol{e}_{x}, \boldsymbol{e}_{y}$, and $\boldsymbol{e}_{z}$ of unity length and mutually orthogonal. The position vector $\boldsymbol{r}=\left(r_{x}, r_{y}, r_{z}\right)=$ $(x, y, z)$ is written as

$\boldsymbol{r}=r_{x} \boldsymbol{e}_{x}+r_{y} \boldsymbol{e}_{y}+r_{z} \boldsymbol{e}_{z}=x \boldsymbol{e}_{x}+y \boldsymbol{e}_{y}+z \boldsymbol{e}_{z}$

Any vector $\boldsymbol{v}$ can be written in terms of its components $v_{i}$ (where for Cartesian co-ordinates $i=x, y, z)$ as

$$
\boldsymbol{v}=\sum_{i=x, y, z} v_{i} \boldsymbol{e}_{i} \equiv v_{i} \boldsymbol{e}_{i} .
$$

In the last equality we have introduced the Einstein summation convention: a repeated index implies a summation over that index (unless noted otherwise). Other examples of this compact, so-called index notation, is the vector scalar product $\boldsymbol{u} \cdot \boldsymbol{v}$, the length $v$ of a vector $\boldsymbol{v}$, and the $i$ th component of the matrix equation $\boldsymbol{u}=M \boldsymbol{v}$,

$$
\begin{gathered}
\boldsymbol{v} \cdot \boldsymbol{u}=v_{i} u_{i}, \\
v=|\boldsymbol{v}|=\sqrt{\boldsymbol{v}^{2}}=\sqrt{\boldsymbol{v} \cdot \boldsymbol{v}}=\sqrt{v_{i} v_{i}}, \\
u_{i}=M_{i j} v_{j} .
\end{gathered}
$$

For the partial derivatives of a given function $F(\boldsymbol{r}, t)$ we use the symbols $\partial_{i}$, with $i=x, y, z$, and $\partial_{t}$,

$$
\partial_{x} F \equiv \frac{\partial F}{\partial x}, \quad \text { and } \quad \partial_{t} F \equiv \frac{\partial F}{\partial t} \text {. }
$$

The vector differential operator nabla $\boldsymbol{\nabla}$ contains the three partial space derivatives $\partial_{i}$. It plays an important role in differential calculus, and it is defined by

$$
\boldsymbol{\nabla} \equiv \boldsymbol{e}_{x} \partial_{x}+\boldsymbol{e}_{y} \partial_{y}+\boldsymbol{e}_{z} \partial_{z}=\boldsymbol{e}_{i} \partial_{i}
$$

The Laplace operator, which appears in numerous partial differential equations in theoretical physics, is just the square of the nabla operator,

$$
\boldsymbol{\nabla} \cdot \boldsymbol{\nabla} \equiv \nabla^{2} \equiv \partial_{i} \partial_{i}
$$

In terms of $\boldsymbol{\nabla}$ the total time derivative of a quantity $F(\boldsymbol{r}(t), t)$ flowing along with the fluid can be written as

$$
\frac{\mathrm{d} F}{\mathrm{~d} t}=\partial_{t} F+\left(\partial_{t} r_{i}\right) \partial_{i} F=\partial_{t} F+(\boldsymbol{v} \cdot \nabla) F .
$$

Since $\boldsymbol{\nabla}$ is a differential operator, the order of the factors does matter in a scalar product containing it. So, whereas $\boldsymbol{v} \cdot \boldsymbol{\nabla}$ in the previous equation is a scalar differential operator, the product $\boldsymbol{\nabla} \cdot \boldsymbol{v}$ with the reversed order of the factors is a scalar function. The latter appears so often in mathematical physics that it has acquired its own name, namely the divergence of the vector field,

$$
\boldsymbol{\nabla} \cdot \boldsymbol{v} \equiv \partial_{x} v_{x}+\partial_{y} v_{y}+\partial_{z} v_{z}=\partial_{i} v_{i}
$$

Concerning integrals, we denote the $3 \mathrm{D}$ integral measure by $\mathrm{d} \boldsymbol{r}$, so that in Cartesian co-ordinates we have $\mathrm{d} \boldsymbol{r}=\mathrm{d} x \mathrm{~d} y \mathrm{~d} z$. We also consider definite integrals as operators acting on integrands, thus we keep the integral sign and the associated integral measure together to the left of the integrand. As an example, the integral in spherical coordinates $(r, \theta, \phi)$ over a spherical body with radius $a$ of the scalar function $S(\boldsymbol{r})$ is written as

$$
\begin{gathered}
\int_{\text {sphere }} \mathrm{d} x \mathrm{~d} y \mathrm{~d} z S(x, y, z)=\int_{\text {sphere }} \mathrm{d} \boldsymbol{r} S(\boldsymbol{r}) \\
=\int_{0}^{a} r^{2} \mathrm{~d} r \int_{0}^{\pi} \sin \theta d \theta \int_{0}^{2 \pi} \mathrm{d} \phi S(r, \theta, \phi) .
\end{gathered}
$$

When working with vectors and tensors it is advantageous to use the following two special symbols: the Kronecker delta $\delta_{\mathrm{ij}}$ and and the Levi-Civita symbol $\varepsilon_{\mathrm{ijk}}$,

$$
\delta_{i j}=\left\{\begin{array}{l}
1, \text { for } i=j, \\
0, \text { for } i \neq j,
\end{array}\right.
$$

$$
\varepsilon_{i j k}=\left\{\begin{array}{c}
+1, \text { if }(i j k) \text { is an even } \\
\text { permutation of }(x y z), \\
-1, \text { if }(i j k) \text { is an odd } \\
\text { permutation of }(x y z), \\
0, \text { otherwise. }
\end{array}\right.
$$

In the index notation, the Levi-Cevita symbol appears directly in the definition of the $i$ th component of the cross-product 
$\boldsymbol{u} \times \boldsymbol{v}$ of two vectors $\boldsymbol{u}$ and $\boldsymbol{v}$, and of the rotation $\boldsymbol{\nabla} \times \boldsymbol{v}$,

$(\boldsymbol{u} \times \boldsymbol{v})_{i} \equiv \varepsilon_{i j k} u_{j} v_{k}$, and $(\boldsymbol{\nabla} \times \boldsymbol{v})_{i} \equiv \varepsilon_{i j k} \partial_{j} v_{k}$.

When evaluating the rotation of a rotation, the "pairing-minus-antipairing" theorem is very useful,

$$
\varepsilon_{i j k} \varepsilon_{i l m}=\delta_{j l} \delta_{k m}-\delta_{j m} \delta_{k l} .
$$

As a last mathematical subject, we mention Gauss's theorem, which we shall employ repeatedly below. For a given vector field $\boldsymbol{V}(\boldsymbol{r})$, it relates the volume integral in a given region $\Omega$ of the divergence $\boldsymbol{\nabla} \cdot \boldsymbol{V}$ to the integral over the surface $\partial \Omega$ of the flux $\boldsymbol{V} \cdot \boldsymbol{n} \mathrm{d} a$ through an area element $\mathrm{d} a$ with the surface normal $\boldsymbol{n}$,

$$
\begin{aligned}
& \int_{\Omega} \mathrm{d} \boldsymbol{r} \nabla \cdot \boldsymbol{V}=\int_{\partial \Omega} \mathrm{d} a \boldsymbol{n} \cdot \boldsymbol{V}, \\
& \text { or } \int_{\Omega} \mathrm{d} \boldsymbol{r} \partial_{j} V_{j}=\int_{\partial \Omega} \mathrm{d} a n_{j} V_{j} .
\end{aligned}
$$

By definition, the surface normal $\boldsymbol{n}$ of a closed surface is an outward-pointing unit vector perpendicular to the surface, hence the name divergence. For a tensor $T_{j k}$ of rank 2 the theorem states $\int_{\Omega} \mathrm{d} \boldsymbol{r} \partial_{j} T_{j k}=\int_{\partial \Omega} \mathrm{d} a n_{j} T_{j k} \quad$ with a straightforward generalization to higher ranks.

\section{Governing equations}

\section{A. The continuity equation}

The first governing equation to be derived is the continuity equation, which expresses the conservation of mass. We consider a compressible fluid, i.e. the density $\rho$ may vary as function of space and time, and a fixed, arbitrarily shaped region $\Omega$ in the fluid as sketched in Fig. 2 . The total mass $M(\Omega, t)$ inside $\Omega$ can be expressed as a volume integral over the density $\rho$,

$$
M(\Omega, t)=\int_{\Omega} \mathrm{d} \boldsymbol{r} \rho(\boldsymbol{r}, t) .
$$

Since mass can neither appear nor disappear spontaneously in non-relativistic mechanics, $M(\Omega, t)$ can only vary due to a mass flux through the surface $\partial \Omega$ of the region $\Omega$. The mass current density $\boldsymbol{J}$ is defined as the mass density $\rho$ times the advection velocity $\boldsymbol{v}$, i.e. the mass flow per oriented area per time $\left(\mathrm{kg} \mathrm{m}^{-2} \mathrm{~s}^{-1}\right)$,

$$
\boldsymbol{J}(\boldsymbol{r}, t)=\rho(\boldsymbol{r}, t) \boldsymbol{v}(\boldsymbol{r}, t) .
$$

As the region $\Omega$ is fixed $\partial_{t} M(\Omega, t)$ can be calculated either by differentiating the volume integral eqn (16),

$$
\begin{aligned}
\partial_{t} M(\Omega, t) & =\partial_{t} \int_{\Omega} \mathrm{d} \boldsymbol{r} \rho(\boldsymbol{r}, t) \\
& =\int_{\Omega} \mathrm{d} \boldsymbol{r} \partial_{t} \rho(\boldsymbol{r}, t),
\end{aligned}
$$

or as a surface integral over $\partial \Omega$ of the mass current density using eqn (17) and Fig. 2,

$$
\begin{aligned}
\partial_{t} M(\Omega, t) & =\int_{\partial \Omega} \mathrm{d} a(-n) \cdot \boldsymbol{J} \\
& =-\int_{\partial \Omega} \mathrm{d} a \boldsymbol{n} \cdot(\rho \boldsymbol{v}) \\
& =-\int_{\Omega} \mathrm{d} \boldsymbol{r} \nabla \cdot[\rho(\boldsymbol{r}, t) \boldsymbol{v}(\boldsymbol{r}, t)] .
\end{aligned}
$$

The last expression is obtained by applying Gauss's theorem eqn (15) to the vector field $\boldsymbol{V} \equiv \boldsymbol{\rho} \boldsymbol{v}$. We have used $-\boldsymbol{n}$ because this is the direction of entering the region. It follows immediately from eqn (18) and (19) that

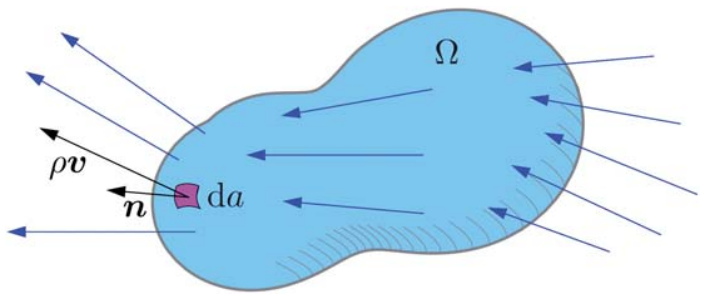

Fig. 2 A sketch of the mass current density field $\rho \boldsymbol{v}$ (long arrows) flowing through an arbitrarily shaped, but fixed region $\Omega$ (blue). Any infinitesimal area d $a$ (purple) is associated with an outwardpointing unit vector $\boldsymbol{n}$ (short arrow) perpendicular to the local surface. The current flowing out through the area $\mathrm{d} a$ is given by $\mathrm{d} a$ times the projection $\rho \boldsymbol{v} \cdot \boldsymbol{n}$ of the current density on the surface unit vector.

$$
\int_{\Omega} \mathrm{d} \boldsymbol{r} \partial_{t} \rho(\boldsymbol{r}, t)=-\int_{\Omega} \mathrm{d} \boldsymbol{r} \nabla \cdot[\rho(\boldsymbol{r}, t) \boldsymbol{v}(\boldsymbol{r}, t)] .
$$

This result is true for any choice of region $\Omega$. However, this is only possible if the integrands are identical. Thus we have derived the continuity equation,

$$
\partial_{t} \rho=-\nabla \cdot(\rho v) \text { or } \partial_{t} \rho=-\partial_{j}\left(\rho v_{j}\right) .
$$

In many cases, especially in microfluidics, where the flow velocity is much smaller than the velocity of speed of sound (pressure waves), the fluid appears incompressible. Thus, $\rho$ is constant in space and time, and the continuity equation simplifies to,

$$
\boldsymbol{\nabla} \cdot \boldsymbol{v}=0 \text { or } \partial_{i} v_{i}=0 .
$$

In Part 2 of the Tutorial Series, ${ }^{37}$ compressibility $\rho=\rho(p)$ will be discussed further in connection with acoustics.

\section{B. The Navier-Stokes equation}

The second governing equation, the Navier-Stokes equation, is the equation of motion for the Eulerian velocity field directly related to momentum conservation and the momentum density $\rho v$. It is derived using an approach similar to that which led us to the continuity equation. We consider the $i$ th component $P_{i}(\Omega, t)$ of the total momentum of the fluid inside an arbitrarily shaped, but fixed, region $\Omega$. In analogy with the mass eqn (18), the rate of change of the momentum is given by

$$
\begin{aligned}
\partial_{t} P_{i} & =\partial_{t} \int_{\Omega} \mathrm{d} \boldsymbol{r} \rho(\boldsymbol{r}, t) v_{i}(\boldsymbol{r}, t) \\
& =\int_{\Omega} \mathrm{d} \boldsymbol{r}\left[\left(\partial_{t} \rho\right) v_{i}+\rho \partial_{t} v_{i}\right] .
\end{aligned}
$$

In contrast to the mass inside $\Omega$, which according to eqn (19) can only change by advection through the surface $\partial \Omega$, the momentum $P_{i}(\Omega, t)$ can change both by advection and by the action of forces given by Newton's second law. These forces can be divided into body forces that act on the interior of $\Omega, e . g$. gravitational and electrical forces, and contact forces that act on the surface $\partial \Omega$ of $\Omega$, e.g. pressure and viscosity forces. Thus, the rate of change of the $i$ th component of the momentum can be written as 


$$
\partial_{t} P_{i}=\partial_{t} P_{i}^{\mathrm{body}}+\partial_{t} P_{i}^{\mathrm{adv}}+\partial_{t} P_{t}^{\mathrm{pres}}+\partial_{t} P_{i}^{\text {visc }} .
$$

A body force $\boldsymbol{f}^{\text {body }}$ is an external force that acts throughout the entire body of the fluid. The change in the momentum of $\Omega$ due to $f^{\text {body }}, e . g$. gravity in terms of the density $\rho$ and the acceleration of gravity $g$, is

$$
\begin{aligned}
\partial_{t} P_{i}^{\text {body }} & =\int_{\Omega} \mathrm{d} \boldsymbol{r} f_{i}^{\text {body }}=\int_{\Omega} \mathrm{d} \boldsymbol{r}(\rho \boldsymbol{g})_{i} \\
& =\int_{\Omega} \mathrm{d} \boldsymbol{r} \rho g_{i} .
\end{aligned}
$$

For the advection of momentum $\rho v$ into $\Omega$, we note that it is described in terms of the tensor $(\rho \boldsymbol{v}) \boldsymbol{v}$, just as advection of density $\rho$ is described by the vector $(\rho) \boldsymbol{v}$. Considering the $i$ th momentum component, we see that the flux of momentum into $\Omega$ through the infinitesimal area $\mathrm{d} a$ is given by $\left(\rho v_{i}\right) \boldsymbol{v} \cdot(-\boldsymbol{n}) \mathrm{d} a$. Thus the total change $\partial_{t} P_{i}^{\text {adv }}(\Omega, t)$ of momentum in $\Omega$ due to advection is

$$
\begin{aligned}
\partial_{t} P_{i}^{\mathrm{adv}} & =\int_{\partial \Omega} \mathrm{d} a(-\boldsymbol{n}) \cdot\left(\rho v_{i} \boldsymbol{v}\right) \\
& =-\int_{\partial \Omega} \mathrm{d} a n_{j} \rho v_{i} v_{j} .
\end{aligned}
$$

The rate of momentum change due to pressure is the sum of the pressure force $p$ $(-\boldsymbol{n}) \mathrm{d} a$ from the surroundings on each infinitesimal area $\mathrm{d} a$ of the surface $\partial \Omega$. Thus, for the $i$ th component of the momentum along the unit vector $\boldsymbol{e}_{i}$ we obtain

$$
\begin{aligned}
\partial_{t} P_{i}^{\text {pres }} & =\int_{\partial \Omega} \mathrm{d} a(-\boldsymbol{n}) \cdot\left(\boldsymbol{p} \boldsymbol{e}_{\boldsymbol{i}}\right) \\
& =-\int_{\partial \Omega} \mathrm{d} a n_{j} p \delta_{i j} .
\end{aligned}
$$

In the last equation we use that $\boldsymbol{n} \cdot \boldsymbol{e}_{i}=$ $n_{j} \delta_{i j}$, whereby $\boldsymbol{n}$ can be ascribed the same free index $j$ different from the momentum component index $i$ as in eqn (26).

The momentum in $\Omega$ is also changed by viscous friction at the surface $\partial \Omega$ from the surrounding fluid. The frictional force $\mathrm{d} \boldsymbol{F}$ on a surface element $\mathrm{d} a$ with the normal vector $\boldsymbol{n}$ must be characterized by a tensor rank of two as two vectors are involved. This tensor is denoted as the viscous stress tensor $\sigma_{i j}^{\prime}$, and it expresses the $i$ th component of the friction force per area acting on a surface element oriented with its surface normal parallel to the $j$ th unit vector $\boldsymbol{e}_{j}$. So we have $\mathrm{d} F_{i}=\sigma_{i j}^{\prime} n_{j} \mathrm{~d} a$, which leads directly to the change in the momentum of $\Omega$ due to the viscous forces at the surface $\partial \Omega$,

$$
\partial_{t} P_{i}^{\text {visc }}(\Omega, t)=\int_{\partial \Omega} \mathrm{d} F_{i}=\int_{\partial \Omega} \mathrm{d} a n_{j} \sigma_{i j}^{\prime} .
$$

The internal friction is only non-zero when fluid particles move relative to each other, hence the viscous stress tensor $\sigma_{i j}^{\prime}$ depends only on the spatial derivatives of the velocity. For the small velocity gradients encountered in microfluidics we can safely assume that only first-order derivatives are relevant. Thus, $\sigma_{i j}^{\prime}$ must thus depend linearly on the velocity gradients $\partial_{i} v_{j}$. Further analysis shows that it must be symmetric, and one way of writing the tensor of rank two satisfying these conditions is ${ }^{4}$

$$
\sigma_{i j}^{\prime}=\eta\left(\partial_{j} v_{i}+\partial_{i} v_{j}\right)+(\beta-1) \eta\left(\partial_{k} v_{k}\right) \delta_{i j},
$$

where the first term relates to the dynamic shear viscosity $\eta$ of an incompressible fluid, and the second term appears when compressibility-induced dilatational viscosity (proportional to $\boldsymbol{\nabla} \cdot \boldsymbol{v}$ ) cannot be neglected. Here, $\beta(\approx 5 / 3$ for water and other simple fluids) is the ratio between the coefficients of the two types of viscosity. The value of $\eta$ is determined experimentally, and for water we have

$$
\begin{aligned}
\eta_{\text {water }}\left(20^{\circ} \mathrm{C}\right) & =1.002 \times 10^{-3} \mathrm{~Pa} \mathrm{~s} \\
& =1.002 \mathrm{mPa} \mathrm{s} .
\end{aligned}
$$

It varies strongly with temperature decreasing from $\eta_{\text {water }}\left(0^{\circ} \mathrm{C}\right)=1.787 \mathrm{mPa} \mathrm{s}$ to $\eta_{\text {water }}\left(100^{\circ} \mathrm{C}\right)=0.282 \mathrm{mPa} \mathrm{s}$.

Combining the results from eqn (23) to (29) yields

$$
\begin{aligned}
& \int_{\Omega} \mathrm{d} \boldsymbol{r}\left[\left(\partial_{t} \rho\right) v_{i}+\rho \partial_{t} v_{i}\right] \\
& =\int_{\partial \Omega} \mathrm{d} a n_{j}\left[-\rho v_{i} v_{j}-p \delta_{i j}+\sigma_{i j}^{\prime}\right] \\
& \quad+\int_{\Omega} \mathrm{d} \boldsymbol{r} \rho g_{i} .
\end{aligned}
$$

Utilizing Gauss's theorem, the surface integral involving $n_{j}$ can be rewritten as a volume integral involving $\partial_{j}$. Since the resulting volume integral equation is valid for any region $\Omega$ the integrands must be identical. After some rewriting we finally arrive at the general equation of motion for the Eulerian velocity field of a viscous fluid,

$$
\rho \partial_{t} v_{i}+\rho v_{j} \partial_{j} v_{i}=-\partial_{i} p+\partial_{j} \sigma_{i j}^{\prime}+\rho g_{i}
$$

The left-hand side can be interpreted as inertial force densities, density times the sum of the local and the advective acceleration, while the right-hand side is the sum of intrinsic or applied force densities. Normally, for the so-called Newtonian fluids at a given temperature, the viscosity coefficients $\eta$ and $\beta$ can be taken as constants, and eqn (32) reduces to the celebrated Navier-Stokes equation for compressible fluids,

$$
\begin{gathered}
\rho\left[\partial_{t} v_{i}+v_{j} \partial_{j} v_{i}\right]= \\
-\partial_{i} p+\eta \partial_{j}^{2} v_{i}+\beta \eta \partial_{i}\left(\partial_{j} v_{j}\right)+\rho g_{i}, \\
\rho\left[\partial_{t} \boldsymbol{v}+(\boldsymbol{v} \cdot \boldsymbol{\nabla}) \boldsymbol{v}\right]= \\
-\boldsymbol{\nabla} p+\eta \nabla^{2} \boldsymbol{v}+\beta \eta \nabla(\boldsymbol{\nabla} \cdot \boldsymbol{v})+\rho \boldsymbol{g} .
\end{gathered}
$$

For incompressible fluids $(\boldsymbol{\nabla} \cdot \boldsymbol{v}=0)$ it becomes

$$
\rho\left[\partial_{t} \boldsymbol{v}+(\boldsymbol{v} \cdot \boldsymbol{\nabla}) \boldsymbol{v}\right]=-\boldsymbol{\nabla} p+\eta \boldsymbol{\nabla}^{2} \boldsymbol{v}+\rho \boldsymbol{g} .
$$

\section{The heat-transfer equation}

The third and last governing equation to be established, is the heat-transfer equation, building on the energy density flux and conservation of energy. The thermodynamic quantities for fluids are usually taken per unit mass, directly relating them to the molecules of the fluid. Thus, we work with the internal energy $\varepsilon$ per unit mass, the entropy $s$ per unit mass, and the volume $1 / \rho$ per unit mass instead of the energy $E$, the entropy $S$, and the volume $v$ of the fluid. The first law of thermodynamics relates internal energy $\mathrm{d} \varepsilon$, heat $T \mathrm{~d} s$, and pressure work $-p \mathrm{~d}(1 / \rho)$, and per unit mass it becomes

$$
d \varepsilon=T \mathrm{~d} s-p \mathrm{~d}\left(\frac{1}{\rho}\right)=T \mathrm{~d} s+\frac{p}{\rho^{2}} \mathrm{~d} \rho .
$$

The densities of the quantities involved are obtained by multiplying them by the mass density $\rho, e . g$. the energy density is written as $\rho \varepsilon$.

In analogy with the mass and momentum densities above, we consider the rate of change $\partial_{t} E(\Omega, t)$ of the energy (power conversion) of the fluid inside some fixed region $\Omega$. As the energy density is given by the sum of the kinetic energy density $\frac{1}{2} \rho v^{2}$ and the internal energy density $\rho \varepsilon$, we obtain 


$$
\begin{aligned}
\partial_{t} E & =\partial_{t} \int_{\Omega} \mathrm{d} \boldsymbol{r}\left[\frac{1}{2} \rho v^{2}+\rho \varepsilon\right] \\
& =\int_{\Omega} \mathrm{d} \boldsymbol{r} \partial_{t}\left[\frac{1}{2} \rho v^{2}+\rho \varepsilon\right] .
\end{aligned}
$$

Here, $E$ can change by energy advection through the surface $\partial \Omega$ of $\Omega$, by work done by pressure and friction forces from the surroundings acting on the surface $\partial \Omega$, and by heat conduction due to thermal gradients at the surface. For simplicity, we disregard heat sources and sinks that could be present inside $\Omega$, so $\partial_{t} E$ can be written as

$$
\partial_{t} E=\partial_{t} E^{\mathrm{adv}}+\partial_{t} E^{\mathrm{pres}}+\partial_{t} E^{\mathrm{visc}}+\partial_{t} E^{\mathrm{cond}} .
$$

The terms $\partial_{t} \rho$ and $\partial_{t} v_{j}$ appearing at the right-hand side of eqn (36) are rewritten using the continuity eqn (21) and the equation of motion eqn (32), whereby

$$
\begin{aligned}
\partial_{t} E= & \int_{\Omega} \mathrm{d} \boldsymbol{r}\left[-\left(\frac{1}{2} v^{2}+\varepsilon\right) \partial_{j}\left(\rho v_{j}\right)\right. \\
& -\rho v_{k} \partial_{k}\left(\frac{1}{2} v^{2}\right)-v_{j} \partial_{j} p+v_{j} \partial_{k} \sigma_{j k}^{\prime} \\
& +\rho \partial_{t} \varepsilon
\end{aligned}
$$

The last term $\rho \partial_{t} \varepsilon$ can be further rewritten by using the first law of thermodynamics (35), thereby bringing the entropy $s$ into play as $\rho \partial_{t} \varepsilon=\rho T \partial_{t} s+(p / \rho) \partial_{t} \rho=\rho T \partial_{t} s-(p / \rho)$ $\partial_{j}\left(\rho v_{j}\right)$. Likewise, the third term is rewritten as $-v_{j} \partial_{j} p=-\rho v_{j} \partial_{j}(\varepsilon+p / \rho)+\rho T v_{j} \partial_{j} s$.

Similar to eqn (19) and (26), the advection of energy into the region is easily expressed in terms of the energy flux density $\boldsymbol{J}_{\varepsilon}=\left(\frac{1}{2} \rho v^{2}+\rho \varepsilon\right) \boldsymbol{v}$,

$$
\begin{aligned}
\partial_{t} E^{\mathrm{adv}} & =\int_{\partial \Omega} \mathrm{d} a(-\boldsymbol{n}) \cdot \boldsymbol{J}_{\varepsilon} \\
& =-\int_{\partial \Omega} \mathrm{d} a n_{j} v_{j}\left[\frac{1}{2} \rho v^{2}+\rho \varepsilon\right] .
\end{aligned}
$$

The power transferred into the region $\Omega$ through the work done by the stress forces due to pressure and viscosity at the surface is given by the product of the stress force vector $\sigma_{j k} n_{j} \mathrm{~d} a$ and the velocity of the fluid $v_{k}$,

$$
\begin{aligned}
& \partial_{t} E^{\mathrm{pres}}+\partial_{t} E^{\mathrm{visc}}= \\
& \int_{\partial \Omega} \mathrm{d} a n_{j}\left[-p \delta_{j k}+\sigma_{j k}^{\prime}\right] v_{k} .
\end{aligned}
$$

Thermal conduction occurs in any medium given a spatially varying temperature field $T(\boldsymbol{r})$. The heat flux density $\boldsymbol{J}_{\text {heat }}$, which is the heat-transfer per area per time given in units of $\mathrm{Jm}^{-2} \mathrm{~s}^{-1}$ or $\mathrm{Wm}^{-2}$, can therefore be expanded in derivatives of the temperature. For small temperature variations only the first derivative $\boldsymbol{\nabla} T$ is significant, and we arrive at Fourier's law of heat conduction for an isotropic medium,

$$
\boldsymbol{J}_{\text {heat }}=-\kappa \boldsymbol{\nabla} T \text {, }
$$

where $\kappa$ is the thermal conductivity of the fluid. For water $\kappa_{\text {water }}=0.597 \mathrm{Wm}^{-1} \mathrm{~K}^{-1}$ at $20^{\circ} \mathrm{C}$. The rate of change of energy by conduction is readily found through the heat flux density and Fourier's law,

$$
\begin{aligned}
\partial_{t} E^{\mathrm{cond}}(\Omega, t) & =\int_{\partial \Omega} \mathrm{d} a(-\boldsymbol{n}) \cdot \boldsymbol{J}_{\text {heat }} \\
& =\int_{\partial \Omega} \mathrm{d} a n_{j}\left(\kappa \partial_{j} T\right) .
\end{aligned}
$$

The heat-transfer equation now follows from eqn (37) using eqn (38), (39), (40), and (42). As before, we use Gauss's theorem to convert the surface integrals into volume integrals, and then equate the integrands to obtain

$$
\begin{gathered}
\rho T\left[\partial_{t} s+v_{j} \partial_{j} s\right]=\sigma_{j k}^{\prime} \partial_{k} v_{j}+\partial_{j}\left[\kappa \partial_{j} T\right], \text { (43a) } \\
\rho T\left[\partial_{t} s+(\boldsymbol{v} \cdot \nabla) s\right]=\boldsymbol{\sigma}^{\prime}: \boldsymbol{\nabla} \boldsymbol{v}+\boldsymbol{\nabla} \cdot(\kappa \boldsymbol{\nabla} T) .
\end{gathered}
$$

The left-hand side is $\rho T$ times the total time derivative of the entropy per unit mass, hence expressing the total gain in heat density per unit time, while the righthand side represents the sources for heat gain: viscous friction and thermal conduction.

In microfluidics, the fluid velocities are generally much smaller than the speed of sound in the fluid. Consequently, pressure variations are minute, leading to the constant pressure approximation, for which $\mathrm{d} s=c_{\mathrm{p}} \mathrm{d} T$, where $c_{\mathrm{p}}$ is the specific heat at constant pressure. In this case the heat-transfer equation reduces to

$\rho c_{\mathrm{p}}\left[\partial_{t} T+\left(v_{j} \partial_{j}\right) T\right]=\partial_{j}\left(\kappa \partial_{j} T\right)+\sigma_{j k}^{\prime} \partial_{k} v_{j} .($

For a fluid at rest $\left(v_{j}=0\right)$ with a constant thermal conductivity $\kappa$, the equation reduces to the Fourier equation,

$$
\partial_{t} T=D_{\mathrm{th}} \nabla^{2} T
$$

which introduces the thermal diffusivity $D_{\mathrm{th}}=\kappa /\left(\rho c_{p}\right)$. For water the measured value is $D_{\text {th }}=1.43 \times 10^{-7} \mathrm{~m}^{2} \mathrm{~s}^{-1}$ at $20{ }^{\circ} \mathrm{C}$.

\section{Flow solutions}

Mathematically, the richness and beauty of hydrodynamic phenomena are spawned by the non-linear term $\rho(\boldsymbol{v} \cdot \boldsymbol{\nabla}) \boldsymbol{v}$ in the Navier-Stokes equation. However, this term is also responsible for complicating the mathematical treatment of the equation; the solutions of the equation have never been fully characterized.

In the following we study two different cases where this complicating non-linear term can be neglected. The first case is the limit of low flow velocities, a limit highly relevant for microfluidic systems. Here, the non-linear term can be neglected as it is much smaller than the viscous term, and we enter the linear regime of the so-called Stokes flow or creeping flow. The second case is when translation symmetry of long, straight channels implies the exact vanishing of the non-linear term. In particular we study the steady-state pressure-driven Poiseuille flow.

\section{A. Hydrostatic pressure}

The simplest case is a fluid in mechanical equilibrium. Such a fluid is at rest relative to the walls of the vessel containing it, and the velocity field is therefore trivially zero everywhere, $\boldsymbol{v}=\mathbf{0}$. If we let $z$-axis point upwards, the gravitational acceleration is $\boldsymbol{g}=-\boldsymbol{g} \boldsymbol{e}_{z}$, and the Navier-Stokes eqn (33b) becomes

$$
\mathbf{0}=-\boldsymbol{\nabla} p_{\mathrm{hs}}-\rho g \boldsymbol{e}_{z},
$$

where the subscript "hs" refers to hydrostatic. For an incompressible fluid, say water, the density $\rho$ is constant and $p_{\mathrm{hs}}$ is easily found to be

$$
p_{\mathrm{hs}}(z)=p^{*}-\rho g z,
$$

where $p^{*}$ is the pressure at the arbitrarily defined zero level $z=0$. In many microfluidic applications this is the only manifestation of gravity. It is therefore customary to write the total pressure as $p_{\text {tot }}=p+p_{\text {hs }}$, such that in the NavierStokes equation the gravitational body force is cancelled by the gradient of hydrostatic pressure. The resulting 
Navier-Stokes equation thus contains the auxiliary pressure $p$ and no gravitational body force.

The hydrostatic pressure $p_{\mathrm{hs}}$ provides an easy way of generating pressure differences in liquids: the pressure at the bottom of a liquid column of height $\Delta H$ is higher by $\Delta p=\rho g \Delta H$ than the pressure at the top. For water $\rho g \approx 10^{4} \mathrm{~Pa} \mathrm{~m}^{-1}$, so a vertical water column of height $10 \mathrm{~cm}$ creates $\Delta p=1 \mathrm{kPa}$, while it takes a height of $10 \mathrm{~m}$ to create $10^{5} \mathrm{~Pa}=1 \mathrm{bar} \approx 1 \mathrm{~atm}$.

\section{B. The Reynolds number and the Stokes equation}

For small flow velocities the non-linear term is expected to be negligible. To determine the condition for this, we nondimensionalize the Navier-Stokes equation: all physical variables are expressed in units of characteristic scales. For a system characterized by only one length scale $L_{0}$ and one velocity scale $V_{0}$, the expression of co-ordinates and velocity in terms of dimensionless co-ordinates and velocity is

$$
\boldsymbol{r}=L_{0} \tilde{\boldsymbol{r}}, \text { and } \boldsymbol{v}=V_{0} \tilde{\boldsymbol{v}},
$$

where the tilde indicates a non-dimensionalized quantity, i.e. a pure number. Once $L_{0}$ and $V_{0}$ have been fixed, the scales $T_{0}$ and $P_{0}$ for time and pressure follow,

$$
t=\frac{L_{0}}{V_{0}} \tilde{t}=T_{0} \tilde{t}, \text { and } p=\frac{\eta V_{0}}{L_{0}} \tilde{p}=P_{0} \tilde{p} \text {. }
$$

Viscosity dominates in microfluidics, so we choose $P_{0}=\eta V_{0} / L_{0}$ and not as $\rho V_{0}^{2}$. By insertion of eqn (48a) and (48b) into the Navier-Stokes eqn (33b) excluding the body-forces as well as the compressibility term, and using the straightforward scaling of the derivatives, $\partial_{t}=\left(1 / T_{0}\right) \tilde{\partial}_{t}$ and $\nabla=\left(1 / L_{0}\right) \tilde{\nabla}$, we obtain

$$
\begin{array}{r}
p\left[\frac{V_{0}}{T_{0}} \tilde{\partial_{t}} \tilde{\boldsymbol{v}}+\frac{V_{0}^{2}}{L_{0}}(\tilde{\boldsymbol{v}} \cdot \tilde{\nabla}) \tilde{\boldsymbol{v}}\right] \\
=-\frac{P_{0}}{L_{0}} \tilde{\nabla} \tilde{p}+\frac{\eta V_{0}}{L_{0}^{2}} \tilde{\nabla}^{2} \tilde{\boldsymbol{v}}
\end{array}
$$

Dividing by $\eta V_{0} / L_{0}^{2}$ this becomes

$$
\operatorname{Re}\left[\tilde{\partial}_{t} \tilde{\boldsymbol{v}}+(\tilde{\boldsymbol{v}} \cdot \tilde{\nabla}) \tilde{\boldsymbol{v}}\right]=-\tilde{\nabla} \tilde{p}+\tilde{\nabla}^{2} \tilde{\boldsymbol{v}}
$$

$$
R e \equiv \frac{\rho V_{0} L_{0}}{\eta}
$$

where the dimensionless parameter $R e$, the so-called Reynolds number, has been introduced.

For $R e \ll 1$ the left-hand side of eqn (50) can be neglected. For water in microfluidics typical values are $\eta / \rho=10^{-6} \mathrm{~m}^{2} \mathrm{~s}^{-1}, L_{0} \approx 10^{-4} \mathrm{~m}$, and $V_{0} \approx 10^{-3} \mathrm{~m} \mathrm{~s}^{-1}$, which leads to a small Reynolds number, $R e \approx 0.1$.

Returning to physical variables in the limit of low Reynolds number, the nonlinear Navier-Stokes equation is reduced to the linear Stokes equation,

$$
\mathbf{0}=-\boldsymbol{\nabla} p+\eta \nabla^{2} \boldsymbol{v},
$$

If the time dependence is controlled by some external time scale different from the intrinsic scale $T_{0}=L_{0} / V_{0}$, the time derivative is not necessarily negligible, and we must employ the time-dependent, linear Stokes equation,

$$
\rho \partial_{t} \boldsymbol{v}=-\boldsymbol{\nabla} p+\eta \nabla^{2} \boldsymbol{v} .
$$

For zero pressure gradient, and introducing the kinematic viscosity $\nu$, it reduces to a diffusion equation

$$
\partial_{t} v=\nu \nabla^{2} v, \quad \text { with } \quad \nu=\frac{\eta}{\rho},
$$

where $\nu$ acts as the diffusivity of momentum in the fluid.

An important example of Stokes flow relates to particle solutions. Let an external force move a spherical particle of radius $a$ with velocity $v_{\mathrm{p}}$ through a fluid that itself moves with velocity $\boldsymbol{v}$ far from the particle. Given the no-slip boundary condition on the particle surface $\partial \Omega_{\mathrm{p}}$, the velocity and pressure field of the fluid can be found from eqn (52), and from that follows the viscous Stokes drag $\boldsymbol{F}_{\text {drag }}$ on the particle, ${ }^{4}$

$$
\begin{aligned}
\boldsymbol{F}_{\mathrm{drag}} & =\int_{\partial \Omega_{\mathrm{p}}} \mathrm{d} a\left(-p \boldsymbol{n}+\sigma^{\prime} \cdot \boldsymbol{n}\right) \\
& =6 \pi \eta a\left(\boldsymbol{v}-\boldsymbol{v}_{\mathrm{p}}\right) .
\end{aligned}
$$

Changing the particle concentration from zero to a few volume percent does not affect the flow profile significantly, while the viscosity may increase by a few percent.

\section{Poiseuille flow}

Our prime example of a solution to the Navier-Stokes equation in the dynamic case, is the pressure-driven, steady-state flow in a straight channel, also known as Poiseuille flow, see Fig. 3. This class of flow is of major importance for the basic understanding of liquid handling in microfluidic lab-on-a-chip systems.

In a Poiseuille flow the fluid is driven through a long, straight, and rigid channel of length $L$ by imposing a pressure difference, $\Delta p$, between the two ends of the channel. The channel is placed horizontally along the $x$-axis, so along the $z$-axis gravity is balanced by the hydrostatic pressure. Furthermore, the crosssection of the channel is constant along the $x$-axis, so the liquid in the channel is only affected by the force from the pressure drop along the $x$-axis. The velocity field is therefore assumed to have only an $x$-component, and this component depends only on the transverse coordinates $y$ and $z$, such that $\boldsymbol{v}=v_{x}(y, z) \boldsymbol{e}_{x}$. This implies $(\boldsymbol{v} \cdot \boldsymbol{\nabla}) \boldsymbol{v}=\left(v_{x} \partial_{x}\right) v_{x}(y, z) \boldsymbol{e}_{x}=\mathbf{0}$, and the non-linear Navier-Stokes equation becomes the linear Stokes equation.

For the velocity field we employ the socalled no-slip boundary condition: On all points on the solid surface $\partial \Omega$ the velocity
Fig. 3 Poiseuille flow of liquid through a straight channel $\Omega$ (blue), where the flow is subject to the no-slip boundary condition on the surface $\partial \Omega$. The channel is translational invariant in the $x$ direction, and it has an arbitrarily shaped cross-section $\mathcal{C}$ (dark blue) in the $y z$-plane. The pressure at $x=0$ (left) is $\Delta p$ higher than at $x=L$ (right).

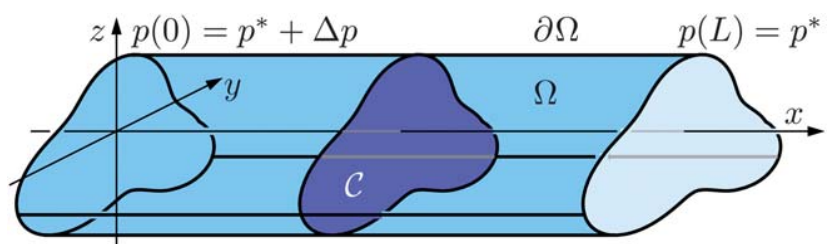


of the fluid equals that of the wall $\boldsymbol{v}_{\text {wall }}$ (often equal to zero),

$$
\boldsymbol{v}(\boldsymbol{r})=\boldsymbol{v}_{\text {wall }}, \text { for } \boldsymbol{r} \in \partial \Omega \text { (no-slip). }
$$

The microscopic origin of this condition is the assumption of complete momentum relaxation between the molecules of the wall and the outermost molecules of the fluid that collide with the wall. The momentum is relaxed on a length scale, which approximately is the molecular mean free path in the fluid, which for liquids means one intermolecular distance ( $\simeq 0.3 \mathrm{~nm}$ ).

The final form of the steady-state Navier-Stokes equation for the Poiseuille flow thus becomes

$$
\begin{gathered}
\boldsymbol{v}(\boldsymbol{r})=v_{x}(y, z) \boldsymbol{e}_{x}, \\
\mathbf{0}=-\boldsymbol{\nabla} p+\eta \nabla^{2}\left[v_{x}(y, z) \boldsymbol{e}_{x}\right] .
\end{gathered}
$$

Since the $y$ and $z$ components of the velocity field are zero, it follows that both $\partial_{y} p$ and $\partial_{z} p$ are zero, and consequently the pressure field depends only on $x, p(r)=p(x)$. Using this result, the $x$ component of the Navier-Stokes eqn (57b) becomes

$$
\eta\left[\partial_{y}^{2}+\partial_{z}^{2}\right] v_{x}(y, z)=\partial_{x} p(x) .
$$

Here, it is seen that the left-hand side is a function of $y$ and $z$, while the right-hand side is a function of $x$. Therefore, the only possible solution is that the two sides of the Navier-Stokes equation equal the same constant. However, a constant pressure gradient $\partial_{x} p(x)$ implies that the pressure must be a linear function of $x$, and using the boundary conditions for the pressure we obtain

$$
p(\boldsymbol{r})=\frac{\Delta p}{L}(L-x)+p^{*} .
$$

With this we arrive at the secondorder partial differential equation for $v_{x}(y, z)$ in the cross-section $\mathcal{C}$ obeying noslip boundary conditions at the solid walls $\partial \Omega$,

$$
\left[\partial_{y}^{2}+\partial_{z}^{2}\right] v_{x}(y, z)=-\frac{\Delta p}{\eta L}, \text { for }(y, z) \in \mathcal{C}
$$

$$
v_{x}(y, z)=0, \text { for }(y, z) \in \partial \Omega .
$$

The resulting velocity field can be determined analytically for a limited

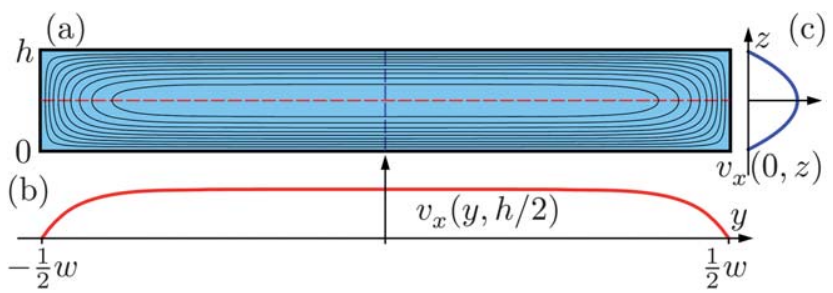

Fig. 4 (a) Contour lines for the velocity field $v_{x}(y, z)$ for a Poiseuille-flow in a rectangular channel. The contour lines are shown in steps of $10 \%$ of the maximal value $v_{x}(0, h / 2)$. (b) A plot (red) of $v_{x}(y, h / 2)$ along the long centerline. (c) A plot (blue) of $v_{x}(0, z)$ along the short centerline.

number of cross-section shapes, as done in ref. 4. Here, we present three of these solutions: a circular cross-section of radius $a$; the space between two horizontal infinite parallel plates placed at $z=0$ and $z=h$; and a rectangular crosssection $-\frac{1}{2} w<y<\frac{1}{2} w$ and $0<z<h$, see Fig. 4(a),

$v_{x}(y, z)=\frac{\Delta p}{4 \eta L}\left(a^{2}-y^{2}-z^{2}\right)$, circular tube,

$v_{x}(y, z)=\frac{\Delta p}{2 \eta L}(h-z) z, \quad$ parallel plates

$v_{x}(y, z)$

$=\frac{4 h^{2} \Delta p}{\pi^{3} \eta L} \sum_{n, \text { odd }}^{\infty} \frac{1}{n^{3}}\left[1-\frac{\cosh \left(n \pi \frac{y}{h}\right)}{\cosh \left(n \pi \frac{w}{2 h}\right)}\right]$ $\sin \left(n \pi \frac{z}{h}\right)$, rectangular cross-section, $h<w$.

Inspection shows that these solutions are correct. Note how the flow profile in the rectangular case is independent of $y$ along a large part $w-h$ of the long width, Fig. 4 (b), but parabolic across the short height, Fig. 4(c).

\section{Flow rate}

Once the velocity field is determined, it is possible to calculate the so-called volumetric flow rate $Q$, which is defined as the fluid volume discharged by the channel per unit time. In the case of the geometry of Fig. 3 we have

$$
Q=\int_{C} \mathrm{~d} y \mathrm{~d} z v_{x}(y, z)=\mathcal{A} v_{\mathrm{avr}}
$$

where $v_{\text {avr }}=(1 / \mathcal{A}) \int_{C} \mathrm{~d} y \mathrm{~d} z v_{x}(y, z)$ is the average velocity and $\mathcal{A}$ is the cross-section area. The flow rate for three selected Poiseuille flows are

$$
Q=\frac{\pi a^{4}}{8 \eta L} \Delta p, \quad \text { circular tube }
$$

$$
Q=\frac{h^{3} w}{12 \eta L} \Delta p, \quad \text { parallel-plates }
$$

$$
Q \approx\left[1-0.630 \frac{h}{w}\right] \frac{h^{3} w}{12 \eta L} \Delta p,
$$

$$
\text { rectangular, } h<w \text {. }
$$

For the Poiseuille flow in a rectangular channel of height $h$ and width $w$, an analytical solution in closed form has not yet been found. However, the error of the approximate result (63c) is just $13 \%$ for the worst case (a square with $h=w$ ), while already for an aspect ratio of a half (a rectangle with $h=w / 2$ ) it has decreased to $0.2 \%$.

The SI unit of flow rate is $\mathrm{m}^{3} \mathrm{~s}^{-1}$, but in microfluidics volume is often measured in $\mu \mathrm{L}=\mathrm{mm}^{3}$ and time in minutes, so the following conversion factors are useful,

$$
1 \frac{\mu \mathrm{L}}{\mathrm{s}}=10^{-9} \frac{\mathrm{m}^{3}}{\mathrm{~s}}, \quad 1 \frac{\mu \mathrm{L}}{\mathrm{min}}=1.67 \times 10^{-11} \frac{\mathrm{m}^{3}}{\mathrm{~s}} .
$$

\section{Equivalent circuit modeling}

Often it suffices to find the flow rate through a given microfluidic network rather than the detailed flow field. To this end the method of equivalent circuit modeling may be used. This type of modeling is only strictly valid for steady Stokes flow in the limit of zero velocity, however, it is a good 
approximation for $R e \leqq 1$ in long, narrow channels, for which entrance effects are negligible, and for changes propagating slower than the speed of sound.

\section{A. Hydraulic resistance}

Above we have found that a constant pressure drop $\Delta p$ results in a constant flow rate $Q$. This result can be summarized in the Hagen-Poiseuille law

$$
\Delta p=R_{\mathrm{hyd}} Q, \quad \text { or } \quad R_{\mathrm{hyd}}=\frac{\Delta p}{Q},
$$

where we have introduced the proportionality factor $R_{\text {hyd }}$ known as the hydraulic resistance; a central concept in characterizing and designing microfluidic channels in lab-on-a-chip systems, see the list in Table 1. The SI units used in the Hagen-Poiseuille law are

$$
\begin{gathered}
{[Q]=\frac{\mathrm{m}^{3}}{\mathrm{~s}}, \quad[\Delta p]=\mathrm{Pa}=\frac{\mathrm{kg}}{\mathrm{m} \mathrm{s}^{2}},} \\
{\left[R_{\mathrm{hyd}}\right]=\frac{\mathrm{Pa} \mathrm{s}}{\mathrm{m}^{3}}=\frac{\mathrm{kg}}{\mathrm{m}^{4} \mathrm{~s}} .}
\end{gathered}
$$

The Hagen-Poiseuille law is analogous to Ohm's law, $\Delta U=R I$, for a wire carrying the electrical current $I$ and having the electrical resistance $R$ and the potential drop $\Delta U$. In hydraulic systems volume is moved and $Q$ is volume per time, while in electric systems charge is moved and $I$ is charge per time. Likewise, $\Delta p$ is energy per volume as $\Delta U$ is energy per charge. Hydraulic power is $Q \Delta P$ (vol/ time $\times$ energy/vol) while electric power is $I \Delta U$ (charge/time $\times$ energy/charge). For low Reynolds numbers fluid flow is described by the linear Stokes equation, and to a good approximation the hydraulic resistances obey the same rules

Table 1 The hydraulic resistance for water flowing through straight channels with different crosssectional shapes. The numerical values are calculated using $\eta=1 \mathrm{mPa} \mathrm{s}, L=1 \mathrm{~m}, a=100 \mu \mathrm{m}, b=33$ $\mu \mathrm{m}, h=100 \mu \mathrm{m}$, and $w=300 \mu \mathrm{m}$. Note that the areas are different

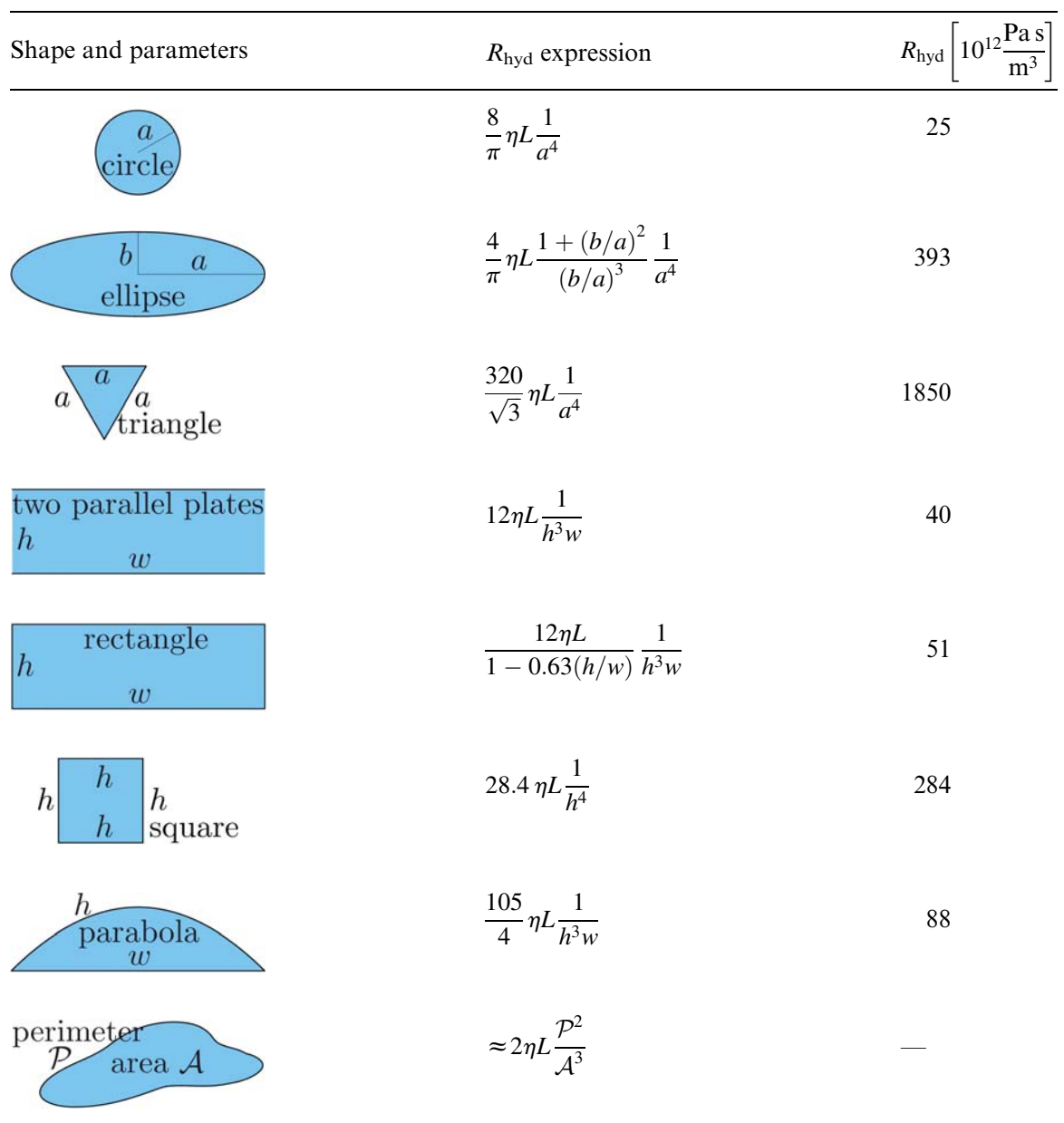

for series and parallel coupling as the electric resistances in linear circuit theory. Thus for two hydraulic resistance $R_{1}$ and $R_{2}$ we have

$$
R_{\text {hyd }}^{\text {series }}=R_{1}+R_{2},
$$

$$
\text { and } R_{\text {hyd }}^{\text {parallel }}=\left(\frac{1}{R_{1}}+\frac{1}{R_{2}}\right)^{-1} .
$$

For a generic fluidic network Kirchhoff's laws apply:

a) The sum of mass flow rates entering/ leaving any node in the circuit is zero. b) The sum of all pressure differences in any closed loop of the circuit is zero.

\section{B. Hydraulic compliance}

The analogy between hydraulic and electric systems can be taken one step further. When the pressure increases by $\Delta p$ in a liquid inside an elastic channel, the volume available to the liquid increases by $\Delta V$. This is analogous to the charging of a capacitor where in increase an voltage by $\Delta U$ increases the charge on the capacitor by $\Delta q=C \Delta U$. The electric capacitance is given by $C=\partial q / \partial U$, and we are led to introduce hydraulic capacitance $C_{\text {hyd, }}$ also known as compliance, given by

$$
C_{\text {hyd }}=\frac{\mathrm{d} V}{\mathrm{~d} p}, \text { with } \quad\left[C_{\text {hyd }}\right]=\mathrm{m}^{3} \mathrm{~Pa}^{-1} \text {. }
$$

As an example of compliance we consider a simple model of a soft-walled channel filled with an incompressible liquid as sketched in Fig. 5(a). If the pressure increases inside the channel, the latter will expand. The compliance $C_{\text {hyd }}$ of the channel is a given constant related to the geometry and the material properties of the channel walls. As a simplification we model the channel as consisting of two subchannels with hydraulic resistances $R_{1}$ and $R_{2}$, respectively, connected in series. The pressure, $p_{\mathrm{c}}$, at the point where the two subchannels connect determines the expansion of the whole channel. The equivalent model is seen in Fig. 5(b). We let the pressure at the inlet be $p^{*}$ for time 
(a)

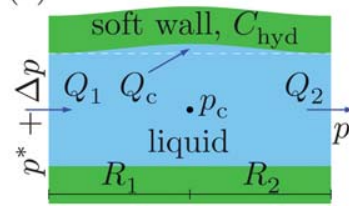

(b)

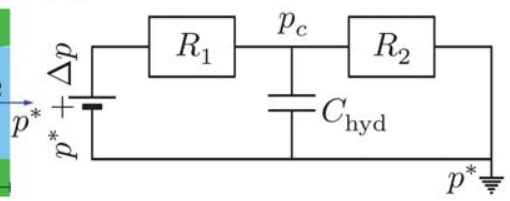

Fig. 5 (a) Compliance in a soft-walled channel (green) filled with liquid (light blue). The liquid center pressure is denoted by $p_{\mathrm{c}}$, while $R_{1}$ and $R_{2}$ denote the hydraulic resistances of the first and second part of the channel, respectively. Mass conservation yields $Q_{1}=Q_{2}+Q_{\mathrm{c}}$. (b) The equivalent circuit diagram corresponding to the soft-walled channel of panel (a), where $R_{1}$ and $R_{2}$ are the hydraulic resistances of each part of the channel, while $C_{\text {hyd }}$ is the compliance of the soft wall. $t<0$ and $p^{*}+\Delta p$ for time $t>0$. The flow rate at the inlet and the outlet are given by the Hagen-Poiseuille law $Q_{1}=$ $\left(p^{*}+\Delta p-p_{\mathrm{c}}\right) / R_{1}$ and $Q_{2}=\left(p_{\mathrm{c}}-p^{*}\right) / R_{2}$, respectively, while the rate of volume expansion inside the chamber is given by $Q_{c}=\partial_{t} V=C_{\mathrm{hyd}} \partial_{t} p_{\mathrm{c}}$. Since the liquid is assumed to be incompressible, conservation of mass leads to $Q_{1}=Q_{2}+Q_{\mathrm{c}}$, and we arrive at a differential equation for the liquid center pressure $p_{\mathrm{c}}$,

$$
\begin{aligned}
\partial_{t} p_{\mathrm{c}}= & -\left(\frac{1}{\tau_{1}}+\frac{1}{\tau_{2}}\right) p_{\mathrm{c}}+\left(\frac{1}{\tau_{1}}+\frac{1}{\tau_{2}}\right) p^{*} \\
& +\frac{1}{\tau_{1}} \Delta p,
\end{aligned}
$$

where $\tau_{1}=R_{1} C_{\text {hyd }}$ and $\tau_{2}=R_{2} C_{\text {hyd }}$ are the hydraulic $R C$ times. The solution,

$$
\begin{aligned}
p_{\mathrm{c}}(t)= & p^{*}+\left(1-\exp \left[\tau_{1}^{-1}+\tau_{2}^{-1}\right] t\right) \\
& \times \frac{\tau_{2}}{\tau_{1}+\tau_{2}} \Delta p,
\end{aligned}
$$

is analogous to the voltage across a capacitor being charged through a voltage divider.

Often the external tubing may lead to long transient times in the external system due to the $R C$-time

$$
\tau_{R C}=R_{\mathrm{hyd}} C_{\mathrm{hyd}}
$$

arising from the hydraulic resistance $R_{\mathrm{hyd}}$ and compliance $C_{\text {hyd }}$. For an elastic tube of fixed length $L$, inner radius $a$, wall thickness $d$, and thickness ratio $\delta=d l a$, $C_{\text {hyd }}$ can be derived from basic theory of elasticity, ${ }^{38}$

$$
C_{\mathrm{hyd}}^{\mathrm{tube}}=2 \pi(1+\bar{\nu}) \frac{(1-2 \bar{\nu})+(1+\delta)^{2}}{(1+\delta)^{2}-1} \frac{a^{2} L}{Y} .
$$

Here, $\bar{\nu}$ is the Poisson ratio, and $Y$ is Young's modulus. Specific examples of transient $R C$ times are listed in Table 2.
If a gas bubble is trapped inside a microfluidic channel, an extra compliance $C_{\text {hyd }}^{\text {buble }}$ is added to the system,

$$
C_{\text {hyd }}^{\text {buble }}=\frac{\mathrm{d} V}{\mathrm{~d} p}=-\frac{\mathrm{d} V^{\text {bubble }}}{\mathrm{d} p}=\frac{4 \pi}{3} \frac{a^{3}}{p^{*}} \text {. }
$$

Here, we have assumed isothermal compression of the gas bubble around ambient pressure $p^{*}$. For isentropic compression the hydraulic compliance is multiplied by the adiabatic factor $\gamma \approx 1.4$. A large bubble with $a=1 \mathrm{~mm}$ leads to $C_{\text {hyd }}^{\text {buble }}=42 \times 10^{-15} \mathrm{~m}^{3} \mathrm{~Pa}^{-1}$, comparable to the silicone tube in Table 2 .

\section{Hydraulic inductance}

The last analogy is inductance. The selfinductance $L_{e l}$, or the electric inertia, is the ability of an electric system to maintain a given current $I$. A rate of change $\partial_{t} I$ in the current induces a potential drop $\Delta U=L_{e l} \partial_{t} I$. Hydraulic inductance $L_{\text {hyd }}$ therefore relates to maintaining an existing volume current $Q=\mathcal{A} v_{\mathrm{avr}}$. Since the rate of change $\partial_{t} Q=\mathcal{A} \partial_{t} v_{\mathrm{avr}}$ involves acceleration, $L_{\mathrm{hyd}}$ must correspond to inertia.

Consider the flow through a channel of length $L$ and cross-section area $\mathcal{A}$. If the force $F$ driving the flow arises from a pressure drop $\Delta p$, we find from Newton's second law that
$\Delta p=F / \mathcal{A}=(\rho L \mathcal{A}) \partial_{t} v_{\mathrm{avr}} / \mathcal{A}$. This leads to $\Delta p=(\rho L / \mathcal{A}) \partial_{t} Q$, from which we can read off the hydraulic inductance $L_{\text {hyd }}$ as

$$
L_{\mathrm{hyd}}=\frac{\rho L}{\mathcal{A}}, \text { with } \quad\left[L_{\mathrm{hyd}}\right]=\frac{\mathrm{Pa} \mathrm{s}^{2}}{\mathrm{~m}^{3}} .
$$

In analogy with the $R C$-time, there is also a transient $R L$-time given by $\tau_{R L}=$ $L_{\text {hyd }} / R_{\text {hyd. }}$. For a tube it is

$$
\tau_{R L}^{\mathrm{tube}}=\frac{L_{\mathrm{hyd}}}{R_{\mathrm{hyd}}}=\frac{\rho a^{2}}{8 \eta}=\frac{a^{2}}{8 \nu},
$$

where in the last equality we have used the kinematic viscosity $\nu$ from eqn (54). Specific examples of $R L$ times are listed in Table 2. For a more complete example of circuit modeling in a microfluidic system with pulsatile flow including compliance and air bubbles see ref. 39 .

\section{Concluding remarks}

In this tutorial paper we have established the theoretical framework for the continuum description of fluids, and based on conservation laws, we have derived the governing equations in microfluidics. Some important, basic flow solutions to the these equations have been presented and analyzed in terms of equivalent circuit modeling, introducing the concepts of hydraulic resistance, compliance, and inductance. The theory presented here does not only apply to the fluid flow, it is also the starting point for the ultrasound acoustics to be treated in the following tutorial paper.

\section{Acknowledgements}

This work was supported by the Danish Council for Independent Research, Technology and Production Sciences, Grant No. 274-09-0342.
Table 2 The hydraulic resistance $R_{\text {hyd }}$, compliance $C_{\text {hyd }}$, and inductance $L_{\text {hyd }}$ as well as the $R C$ and $R L$ times $\left(\tau_{R C}\right.$ and $\left.\tau_{R L}\right)$ for water $\left(\eta=1 \mathrm{~m} \mathrm{~Pa} \mathrm{~s}, \rho=998 \mathrm{~kg} \mathrm{~m}^{-3}\right)$ in a straight circular channel (length $L=1 \mathrm{~m}$, radius $a=0.1 \mathrm{~mm}$, thickness ratio $\delta=1)$ made of either soft silicone rubber ( $Y=2.1 \mathrm{MPa}$, $\bar{\nu}=0.49)$ or hard teflon $(Y=0.5 \mathrm{GPa}, \bar{\nu}=0.45)$. It takes $0.7 \mathrm{~ms}$ for a pressure wave to propagate through the channel

\begin{tabular}{llcccc}
\hline Material & $R_{\text {hyd }}\left[10^{12} \frac{\mathrm{Pas}}{\mathrm{m}^{3}}\right]$ & $C_{\text {hyd }}\left[10^{-15} \frac{\mathrm{m}^{3}}{\mathrm{~Pa}}\right]$ & $L_{\mathrm{hyd}}\left[10^{9} \frac{\mathrm{Pa} \mathrm{s}^{2}}{\mathrm{~m}^{3}}\right]$ & $\tau_{R C}[\mathrm{~ms}]$ & $\tau_{R L}[\mathrm{~ms}]$ \\
\hline Silicone & 25 & 60 & 32 & 1500 & 1.3 \\
Teflon & 25 & 0.25 & 32 & 6.2 & 1.3 \\
\hline
\end{tabular}




\section{References}

1 P. Tabeling, Introduction to Microfluidics, Oxford University Press, Oxford, 2005.

2 G. Karniadakis, A. Beskok and N. Aluru, Microflows and Nanoflows: Fundamentals and Simulation, Springer Science + Business Media, Inc., New York NY, 2005.

3 N.-T. Nguyen and S. T. Wereley, Fundamentals and Applications of Microfluidics, Artech House, Norwood MA, 2nd edn, 2006.

4 H. Bruus, Theoretical Microfluidics, Oxford University Press, Oxford, 2008.

5 J. Berthier and P. Silberzan, Microfluidics for Biotechnology, Artech House, Norwood MA, 2nd edn, 2010.

6 B. Kirby, Micro- and Nanoscale Fluid Mechanics: Transport in Microfluidic Devices, Cambridge University Press, New York, 2010.

7 A. J. Mach and D. Di Carlo, Biotechnol. Bioeng., 2010, 107, 302-311.

8 S. Jacobson, R. Hergenroder, L. Koutny and J. Ramsey, Anal. Chem., 1994, 66, 1114-1118.

9 P. R. C. Gascoyne, J. Noshari, T. J. Anderson and F. F. Becker, Electrophoresis, 2009, 30, 1388-1398.

10 M. Gijs, Microfluidics Nanofluidics, 2004, 1 , 22-40.

11 L. Huang, E. Cox, R. Austin and J. Sturm, Science, 2004, 304, 987-990.

12 D. Johnson and D. Feke, Sep. Technol., 1995, 5, 251-258.

13 K. Yasuda, S. Umemura and K. Takeda, Jpn. J. Appl. Phys., 1995, 34, 2715-2720.

14 J. J. Hawkes and W. T. Coakley, Sens. Actuators, B, 2001, 75, 213-222.

15 J. J. Hawkes, W. T. Coakley, M. Groschl, E. Benes, S. Armstrong, P. J. Tasker and
H. Nowotny, J. Acoust. Soc. Am., 2002, 111, 1259-1266.

16 N. R. Harris, M. Hill, S. Beeby, Y. Shen, N. M. White, J. J. Hawkes and W. T. Coakley, Sens. Actuators, B, 2003, 95, 425-434.

17 F. Petersson, A. Nilsson, C. Holm, H. Jönsson and T. Laurell, Analyst, 2004, 129, 938-43.

18 F. Petersson, A. Nilsson, C. Holm, H. Jönsson and T. Laurell, Lab Chip, $2005, \mathbf{5}, 20-2$.

19 J. Hultström, O. Manneberg, K. Dopf, H. M. Hertz, H. Brismar and M. Wiklund, Ultrasound Med. Biol., 2007, 33, 145-151.

20 M. Evander, L. Johansson, T. Lilliehorn, J. Piskur, M. Lindvall, S. Johansson, M. Almqvist, T. Laurell and J. Nilsson, Anal. Chem., 2007, 79, 2984-2991.

21 J. Svennebring, O. Manneberg, P. SkaftePedersen, H. Bruus and M. Wiklund, Biotechnol. Bioeng., 2009, 103, 323-328.

22 A. Lenshof, A. Ahmad-Tajudin, K. Jaras, A.-M. Sward-Nilsson, L. Aberg, G. Marko-Varga, J. Malm, H. Lilja and T. Laurell, Anal. Chem., 2009, 81, 60306037.

23 J. V. Norris, M. Evander, K. M. HorsmanHall, J. Nilsson, T. Laurell and J. P. Landers, Anal. Chem., 2009, 81, 6089-6095.

24 C. Grenvall, P. Augustsson, J. R. Folkenberg and T. Laurell, Anal. Chem., 2009, 81, 6195-6200.

25 T. Franke, S. Braunmueller, L. Schmid, A. Wixforth and D. A. Weitz, Lab Chip, 2010, 10, 789-794.

26 P. Thevoz, J. D. Adams, H. Shea, H. Bruus and H. T. Soh, Anal. Chem., 2010, 82, 3094 3098.
27 P. Augustsson, R. Barnkob, C. Grenvall, T. Deierborg, P. Brundin, H. Bruus and T. Laurell, Proc. 14th MicroTAS, 3-7 October 2010, Groningen, The Netherlands, 2010, pp. 1337-39.

28 S. M. Hagsäter, T. G. Jensen, H. Bruus and J. P. Kutter, Lab Chip, 2007, 7, 1336-1344.

29 J. Shi, H. Huang, Z. Stratton, Y. Huang and T. J. Huang, Lab Chip, 2009, 9, 3354 3359.

30 O. Manneberg, S. M. Hagsäter, J. Svennebring, H. M. Hertz, J. P. Kutter, $\mathrm{H}$. Bruus and M. Wiklund, Ultrasonics, 2009, 49, 112-119.

31 O. Manneberg, B. Vanherberghen, B. Onfelt and M. Wiklund, Lab Chip, 2009, 9, 833-837.

32 P. Glynne-Jones, R. J. Boltryk, N. R. Harris, A. W. J. Cranny and M. Hill, Ultrasonics, 2010, 50, 68-75.

33 J. D. Adams, P. Thevoz, H. Bruus and H. T. Soh, Appl. Phys. Lett., 2009, 95, 254103-1.

34 S. Oberti, D. Moeller, A. Neild, J. Dual, F. Beyeler, B. J. Nelson and S. Gutmann, Ultrasonics, 2010, 50, 247-257.

35 R. Barnkob, P. Augustsson, T. Laurell and H. Bruus, Lab Chip, 2010, 10, 563-570.

36 P. Augustsson, R. Barnkob, S. T. Wereley, H. Bruus and T. Laurell, Lab Chip, 2011, DOI: $10.1039 / \mathrm{c} 1 \mathrm{lc} 20637 \mathrm{k}$.

37 H. Bruus, Lab Chip, 2011, DOI: 10.1039/ c1lc20770a.

38 L. D. Landau and E. M. Lifshitz, Theory of Elasticity, 3rd Edition. Course of Theoretical Physics, Pergamon Press, Oxford, 1986, vol. 7.

39 S. Vedel, L. H. Olesen and H. Bruus, J. Micromech. Microeng., 2010, 20, 035026 . 